\title{
BMJ Open International observational atopic dermatitis cohort to follow natural history and treatment course: TARGET- DERM AD study design and rationale
}

\author{
Katrina Abuabara (D) , Jonathan I Silverberg (D) , ${ }^{2}$ Eric L Simpson (D) , ${ }^{3}$ \\ Amy S Paller (D) , ${ }^{4}$ Lawrence F. Eichenfield (D) , ${ }^{5}$ Robert Bissonnette (D) , \\ James Krueger (D) , ${ }^{7}$ John E. Harris (D) , ${ }^{8}$ Laura Dalfonso (D) ${ }^{9}$ \\ Stephanie E Watkins, ${ }^{10}$ Julie M Crawford (D) , ${ }^{10}$ D Thaçi (D) ,11 \\ Emma Guttman-Yassky (D) ${ }^{12}$
}

To cite: Abuabara K, Silverberg JI, Simpson EL, et al. International observational atopic dermatitis cohort to follow natural history and treatment course: TARGETDERM AD study design and rationale. BMJ Open 2020;10:e039928. doi:10.1136/ bmjopen-2020-039928

- Prepublication history and additional materials for this paper is available online. To view these files, please visit the journal online (http://dx.doi org/10.1136/bmjopen-2020039928).

Received 17 May 2020 Revised 04 October 2020 Accepted 07 October 2020
Check for updates

(C) Author(s) (or their employer(s)) 2020. Re-use permitted under CC BY-NC. No commercial re-use. See rights and permissions. Published by BMJ.

For numbered affiliations see end of article.

Correspondence to Dr Katrina Abuabara; katrina.abuabara@ucsf.edu

\section{ABSTRACT}

Introduction As new topical and systemic treatments become available for atopic dermatitis (AD), there is a need to understand how treatments are being used in routine clinical practice, their comparative effectiveness and their long-term safety in diverse clinical settings.

Methods and analysis The TARGET-DERM AD cohort is a Iongitudinal, observational study of patients with $A D$ of all ages, designed to provide practical information on longterm effectiveness and safety unobtainable in traditional registration trials. Patients with physician-diagnosed $A D$ receiving prescription treatment (topical or systemic) will be enrolled at academic and community clinical centres. Up to 3 years of retrospective medical records, 5 years of prospective medical records, and optional biological samples and patient-reported outcomes will be collected. The primary aims include characterisation of $A D$ treatment regimens, evaluation of response to therapy, and description of adverse events.

Ethics and dissemination TARGET-DERM has been approved by a central IRB (Copernicus Group IRB, 5000 Centregreen Way Suite 200, Cary, North Carolina 27513) as well as local and institutional IRBs. No additional Ethics Committee reviews. Results will be reviewed by a publications committee and submitted to peer-reviewed journals.

Trial registration number NCT03661866, pre-results.

\section{BACKGROUND}

Atopic dermatitis is, by definition, a chronic condition that waxes and wanes over time. ${ }^{1}$ Defects of the epidermal barrier, immune dysregulation and environmental factors are thought to affect disease expression, and it is now recognised as a lifelong disposition with variable clinical manifestations. ${ }^{2}{ }^{3}$ Despite being one of the leading contributors to non-fatal disease burden worldwide, very little is understood about long-term disease control. ${ }^{45}$

\section{Strengths and limitations of this study}

- TARGET-DERM atopic dermatitis is a longitudinal cohort study of patients with atopic dermatitis, including up to 3 years of retrospective data and 5 years of prospective data, allowing for assessment of changes over time in individual patients.

- The study has broad inclusion criteria to allow for capture of patient populations that may be underrepresented in clinical trials, and inclusion of patients from both academic and community practices will improve generalisability.

- The study relies on standardised extraction from routinely collected medical records.

- The study collects validated investigator global assessments and quarterly patient-reported outcome measures.

- The study has a biospecimen collection.

Pragmatic, real-world studies that capture long-term variability in atopic dermatitis disease activity and management are needed and can provide complementary data to clinical trials. Based on growing knowledge of immune pathways, the pharmacological options for management of atopic dermatitis have expanded over the past several years, with further expansion expected during the next decade. ${ }^{6}$ Clinical trials are often performed in highly selected, adherent study participants who lack significant comorbidities beyond the disease in question. These trials are a good measure of clinical efficacy, but the more germane question for clinicians and their patients is one of real-world effectiveness. Indeed, participants enrolled in dermatology clinical trials may not be highly representative of the general atopic dermatitis population. ${ }^{7-9}$ Subgroups that might be excluded or under-represented in clinical 
trials include racial/ethnic minority and socioeconomic groups, paediatric and geriatric populations, individuals with multiple comorbidities and pregnant women. Additionally, since most trials make comparisons between active ingredients and placebos, more data from direct comparisons between therapies (both new and conventional) are needed. ${ }^{10}$

The overarching objective of the TARGET-DERM AD cohort is to understand the comparative effectiveness and long-term safety of atopic dermatitis treatments. The cohort will also serve as a core resource to understand realworld treatment patterns and disease comorbidities, and to perform translational studies using longitudinal clinical data linked to patient-reported outcomes data and biospecimens. The ultimate goal is to improve patient care for AD. TARGET-DERM is a cooperative consortium of principal investigators from academic institutions and community sites. It is based on a successful model from prior TARGET studies used to elucidate the natural history of disease and comparative effectiveness for several gastrointestinal diseases, starting with hepatitis C. ${ }^{11}$

\section{METHODS AND ANALYSIS}

\section{Study design}

TARGET-DERM AD is a longitudinal, observational cohort study of atopic dermatitis. The primary aims of TARGET-DERM include characterisation of treatment regimens, evaluation of patient outcomes and description of adverse events. Secondary aims include evaluation of the relationship between atopic dermatitis and comorbid medical conditions. No specific treatments will be dictated by enrolment into the study; patient management will follow each site's local standard of care.

Adult and paediatric patients of all ages will be enrolled from up to 100 practices, including both academic practices affiliated with a university health system and community-based or private practice clinical centres. The first patient was enrolled on 25 January 2019 and, as of March 2020, there are 34 active sites and an additional 10 in the start-up phase (figure 1). Site recruitment is ongoing. Enrolment rates by site type, geography, patient

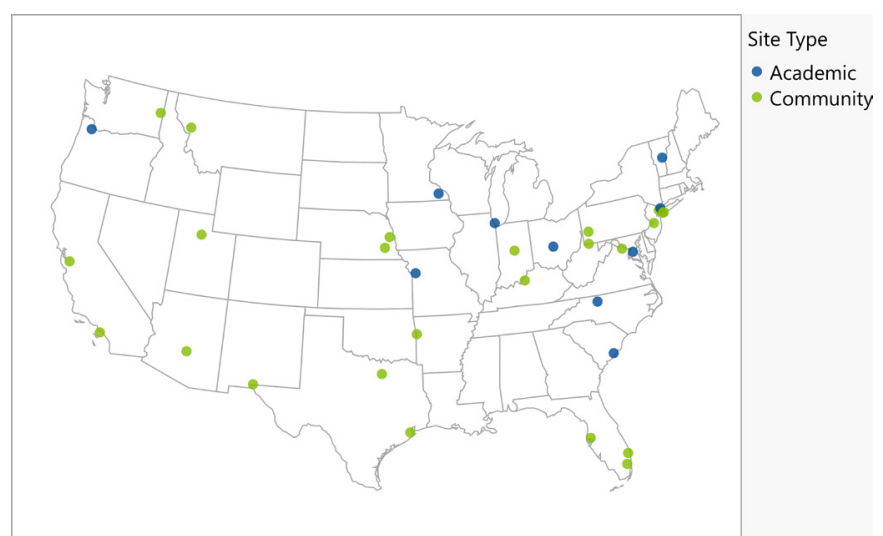

Figure 1 TARGET-DERM has active community (24) and academic (10) sites across the USA. age, patient race/ethnicity, socioeconomic status and type of treatment (topical vs systemic) are reviewed by the academic steering committee monthly and compared with national statistics. The recruitment strategy is designed to be adaptive to ensure adequate cohort diversity.

\section{Study participants}

As this study seeks to reflect real-world clinical practice, there are minimal inclusion/exclusion criteria. Patients of all ages being managed or treated for atopic dermatitis with at least one prescription treatment will be enrolled. A diagnosis of atopic dermatitis made by the treating physician, as in standard clinical practice, will be required for enrolment. During site orientation, diagnostic recommendations from the American Academy of Dermatology will be reviewed with enrolling physicians, and participants will be asked UK Working Party criteria at enrolment to enable post-hoc evaluations based on these diagnostic criteria. ${ }^{12} 13$ Patients will be excluded if they are unable to provide written informed consent or assent, or are participating in an investigational study of a systemic treatment for atopic dermatitis at the time of enrolment. Concurrent participation in investigational studies of topical treatments or other registries or observational studies on atopic dermatitis treatment outcomes, however, are permitted.

\section{Patient and public involvement}

At the time of consent, patients in TARGET-DERM are presented with joining this study as a way to advance research in their disease area. In addition, a non-profit medical specialty society dedicated to education and research on atopic dermatitis, the International Eczema Council, has joined the steering committee. Patients were not directly involved with the development or conduct of the study. The plan is to share study results through publication; posters and manuscripts will be displayed on the TARGET website.

\section{Study procedures}

After informed consent is obtained and eligibility confirmed, baseline demographic and background information will be collected. On enrolment and during each follow-up visit for atopic dermatitis, clinicians will record a validated investigator global assessment (vIGA-AD) score. ${ }^{14} \mathrm{Up}$ to 3 years of retrospective medical records, and up to 5 years of prospective medical records will be collected biannually. Records will include clinic notes, hospitalisations, laboratory reports, telephone contact reports, medication lists, biopsy results and imaging. Structured variables to be extracted from these records include demographic factors, personal and family medical history, AD disease characteristics, current medications, laboratory tests and detailed AD treatment history (box 1). There are no study-mandated interventions planned.

Participants will be asked to complete patient-reported outcome (PRO) assessments at baseline and every 
Box 1 Categories for structured clinical record data extraction

- Demographic information: sex at birth, age, race/ethnic group, employment status, country of origin, insurance type.

- Atopic dermatitis (AD) characteristics: age of onset, standardised vIGA-AD score*, total body surface area affected.

- Additional medical history: medical diagnoses (historical and new), pregnancies (with due date and pregnancy outcome), number/ relation of relatives with history of $A D$, number/relation of family with history of allergic diseases (allergic rhinitis, asthma, hay fever), personal history of other immune-mediated inflammatory skin conditions,

- Substance use: alcohol, tobacco, vaping use and frequency, marijuana use, other recreational drug history.

- Current medications: concomitant medications with reasons for medications.

- Clinical/laboratory measures: height, weight, white blood cell, neutrophils, lymphocytes, haemoglobin, platelet count, serum alanine aminotransferase, aspartate transaminase, gammaglutamyltransferase, total bilirubin, direct bilirubin, indirect bilirubin, albumin, creatinine, glucose, calcium, magnesium, phosphorus, potassium, sodium, urea nitrogen, creatine kinase, low-density lipoprotein, high-density lipoprotein, triglycerides, total cholesterol, other labs.

- Procedures: allergy testing, skin biopsies (with type, reason for biopsy, location and pathology report if available), other procedures with indication and date.

- $\mathrm{AD}$ treatment details: current and prior systemic therapy/medication name with start/stop/treatment vehicle, topical therapy/medication name with start/stop/treatment vehicle, phototherapy type with start/stop, dose changes/interruptions/discontinuations, reason for dose changes/interruptions/discontinuations.

- AD treatment safety: adverse events, serious adverse events, alternative treatments used (type, treatment vehicle, concentration).

${ }^{*}$ Note that as part of the study protocol, clinicians are asked to perform a standardised vIGA-AD at each standard of care visit.

3 months thereafter. These address itch, pain and sleep, quality of life, severity, work productivity and activity impairment, tailored to adult or paediatric populations (table 1). PROs were chosen based on Harmonizing Outcome Measures in Eczema clinical practice set recommendations, ${ }^{15}$ and core domains for participants on systemic and phototherapies will include those recommended by the international treatment of atopic dermatitis registry task force (TREAT) eDelphi exercise. ${ }^{16}$

Optional biological samples will be collected yearly for future translational research on genetic and immmunophenotypes and include blood, cutaneous tape strips and saliva. Participants will consent to each optional specimen separately. When possible, blood samples will be collected during regular clinical blood work.

The TARGET steering committee (currently composed of the academic coauthors on this manuscript) developed the study protocol and research plan and gave input on site selection and database design. The study is privately funded by TARGET PharmaSolutions and can be used to provide postauthorisation safety data as requested by licensing and regulatory bodies. TARGET stakeholders, including steering committee members, industry partners, regulatory representatives, and a nonprofit medical specialty society participate in steering committee meetings to drive the research agenda for TARGET studies. The steering committee has oversight over any public dissemination of the data generated in this study.

\section{Data management}

Participating investigational sites provide medical records (including structured and unstructured data) from consented participants. All data will be processed and stored centrally via an electronic data capture system maintained by TARGET PharmaSolutions. Biosamples will be collected at the sites, sent for storage at a central biorepository and tracked in the electronic data capture system by TARGET; investigators and stakeholders can apply to use the samples for future research. Coding of data will be performed using standardised data extraction forms and MedDRA ${ }^{17}$ and WHODrug $^{18}$ dictionaries. Data will be monitored for quality and missingness. Specific efforts will be made to investigate and document any reasons leading to premature study termination. Adverse events will be evaluated bi-annually and reported to regulatory bodies as outlined in the study safety monitoring plan.

\section{Study outcomes}

The primary study outcomes are response to therapy and adverse events. Response to therapy will be based on changes in vIGA-AD as collected during standard care visits and change in patient-reported outcomes collected quarterly. Changes in therapy (ie, discontinuation, stepping-up, or stepping down) will also be considered. Adverse events will be defined as any untoward medical occurrence which deviates from, or is an exacerbation of, the subject's medical history after the time of consent. Additional secondary outcomes include the occurrence and impact of comorbid medical conditions on treatment regimens and vice versa.

\section{Sample size}

At least 4000 study participants from up to 100 clinical centres will be recruited for TARGET-DERM- AD. The sample size was chosen based on logistical considerations and the ability to address multiple study objectives; post-hoc calculations suggest that a sample size of 4000 AD participants (assuming 2000 per group) would enable detection of effect sizes as small as 0.07 for changes in the primary outcome of vIGA-AD.

\section{DISCUSSION}

TARGET-DERM AD is designed to enable the study of real-world treatment patterns and long-term outcomes in atopic dermatitis, including both comparative effectiveness and safety endpoints. The study has broad inclusion criteria to allow for capture of patient populations that may be underrepresented in clinical trials, 
Table 1 Study measures and timing

\begin{tabular}{|c|c|c|c|c|c|c|c|c|c|c|c|c|}
\hline \multirow[b]{3}{*}{ Activity } & \multirow[b]{3}{*}{$\begin{array}{l}\text { Prior } 3 \\
\text { years* }\end{array}$} & \multirow{3}{*}{$\begin{array}{l}\text { Screening/ } \\
\text { enrolment visit } \\
\text { Month } 0\end{array}$} & \multicolumn{10}{|c|}{ Follow-up } \\
\hline & & & \multicolumn{2}{|l|}{ Year 1} & \multicolumn{2}{|l|}{ Year 2} & \multicolumn{2}{|l|}{ Year 3} & \multicolumn{2}{|l|}{ Year 4} & \multicolumn{2}{|l|}{ Year 5} \\
\hline & & & $\begin{array}{l}\text { Month } \\
6\end{array}$ & $\begin{array}{l}\text { Month } \\
12\end{array}$ & $\begin{array}{l}\text { Month } \\
18\end{array}$ & $\begin{array}{l}\text { Month } \\
24\end{array}$ & $\begin{array}{l}\text { Month } \\
30\end{array}$ & $\begin{array}{l}\text { Month } \\
36\end{array}$ & $\begin{array}{l}\text { Month } \\
42\end{array}$ & $\begin{array}{l}\text { Month } \\
48\end{array}$ & $\begin{array}{l}\text { Month } \\
54\end{array}$ & $\begin{array}{l}\text { Month } \\
60\end{array}$ \\
\hline Informed consent & & $x$ & & & & & & & & & & \\
\hline Inclusion/exclusion & & $x$ & & & & & & & & & & \\
\hline $\begin{array}{l}\text { Biologic sample } \\
\text { collection }\end{array}$ & & $x$ & & & & & & & & & & \\
\hline $\begin{array}{l}\text { Patient reported } \\
\text { outcome surveyst }\end{array}$ & & $x$ & Patient & -reportec & d outcom & es will be & complet & ted every & 3 month & & & \\
\hline $\begin{array}{l}\text { Investigator global } \\
\text { assessment† }\end{array}$ & & $X$ & To be c & ollected & at all star & hdard of & care visit & & & & & \\
\hline
\end{tabular}

For paediatrics, these include: UKWPC, ${ }^{12} 26$ POEM-Paediatric/Proxy, ${ }^{27}$ PROMIS-Itch Severity, NRS-Pain, NRS-Sleep, PROMIS-Paediatric Anxiety, PROMIS-Paediatric Depression, Children's Dermatology Life Quality Index (CDLQI) ${ }^{28}$ and PO-SCORAD; these are all completed every 3 months except for UKWPC (one time) and POEM-Paediatric/Proxy, CDLQI and PO-SCORAD (every 6 months).

*Three years of historical records will be submitted following the screening/enrolment visit. During the follow-up period, medical records will be submitted every 6 months for up to 5 years. The first submission during the follow-up period will be 6 months following the screening/enrolment visit. Additional interim medical records submissions may be requested.

†UK Working Party diagnostic criteria (UKWPC), ${ }^{12} 26$ Patient-Oriented Eczema Measure (POEM) ${ }^{29}$ Patient-Reported Outcomes Measurement Information System (PROMIS)-Itch Severity, ${ }^{30}$ Numerical Rating Scale for Pain (NRS-Pain), ${ }^{31}$ NRS-Sleep, ${ }^{32} 33$ PROMISGeneral, PROMIS-Mood and Sleep, PROMIS-Activity and Clothing, PROMIS-Scratching Behaviour, PROMIS-Anxiety, PROMISDepression, PROMIS-Itch Triggers, PROMIS-Itch Quality, ${ }^{34}$ Dermatology Life Quality Index (DLQI), ${ }^{35}$ Patient-Oriented-SCORing Atopic Dermatitis (PO-SCORAD) ${ }^{36}$ and the Work Productivity and Activity Impairment (WPAI). ${ }^{38}$ These are all completed every 3 months except for UKWPC, PROMIS-Itch Triggers and PROMIS-Itch Quality (one time), and DLQI and WPAI (every 6 months).

$\ddagger$ Validated Investigator Global Assessment (VIGA) \pm body surface area assessment will be obtained at baseline and all follow-up visits.

and recruitment of participants from a diverse group of academic and community practices will improve generalisability. TARGET-DERM AD fills an important niche; although other atopic dermatitis registries exist, these are largely focused on the impacts of specific treatments (Pediatric Eczema Elective Registry (PEER) and A Prospective Pediatric Longitudinal Evaluation to Assess the Long-Term Safety of Tacrolimus Ointment for hte Treatment of Atopic Dermatitis (APPLE)), ${ }^{19} 20$ skin infections associated with AD (Atopic Dermatitis Research Network), ${ }^{21}$ or systemic treatments (TREAT).$^{22}$

An important limitation is documentation of atopic dermatitis disease activity, which could be prone to inaccuracies when relying on real-world assessments. In particular, the frequency of patient visits and details in routinely collected medical records may not accurately capture intermittent disease activity and severity. Regular quarterly patient reported assessments and biosamples will enhance the internal validity for the subset of participants consenting to these additional outcomes.

TARGET-DERM AD will serve as the prototype for a larger cohort study of immune-mediated inflammatory skin conditions (IMISC), to ultimately include additional participants with psoriasis, hidradenitis suppurativa, vitiligo, and alopecia areata, and to enrol up to 15000 participants. Although multiple registries and cohorts for psoriasis exist, ${ }^{23-25}$ such data sources are limited for other inflammatory skin diseases. Given the overlap in risk factors and treatment options for IMISC, a joint registry will benefit the growing understanding of these diseases, including the natural history, heterogeneity of different patient subpopulations, real-world management strategies and response to treatment. Additionally, ongoing monitoring of side effect profiles of emerging therapeutic agents used for multiple indications will enhance knowledge of the safety profile. Furthermore, the availability of an established cohesive research network allows nimble responses to investigations of new treatment paradigms with existing agents, as well as future generations of IMISC therapies.

\section{ETHICS AND DISSEMINATION}

The protocol was approved by a central institutional review board (Copernicus IRB), and site-specific IRB approvals are obtained prior to patient enrolment where required (see online supplemental file 1). Results will be published in peer-reviewed journals and presented at national and international meetings and reported in accordance with the Strengthening the Reporting of Observational Studies in Epidemiology recommendations.

Author affiliations

${ }^{1}$ Department of Dermatology, University of California, San Francisco, San Francisco, CA, USA 
${ }^{2}$ Department of Dermatology, George Washington University, Washington, DC, USA ${ }^{3}$ Department of Dermatology, Oregon Health \& Science University, Portland, Oregon, USA

${ }^{4}$ Department of Dermatology, Northwestern University, Chicago, Illinois, USA ${ }^{5}$ Department of Dermatology, University of California, San Diego School, La Jolla, California, USA

${ }^{6}$ Innovaderm Research, Montreal, Quebec, Canada

${ }^{7}$ Department of Immunology, Virology and Microbiology, Rockefeller Institute for Medical Research, New York, New York, USA

${ }^{8}$ Department of Dermatology, University of Massachusetts Medical School, Worcester, MA, USA

${ }^{9}$ Clinical Operations, TARGET PharmaSolutions, Durham, North Carolina, USA ${ }^{10}$ Scientific and Medical Affairs, TARGET PharmaSolutions, Durham, North Carolina, USA

${ }^{11}$ Comprehensive Center for Inflammation Medicine, University of Lubeck, Lubeck, Schleswig-Holstein, Germany

${ }^{12}$ Department of Dermatology, Icahn School of Medicine at Mount Sinai, New York, New York, USA

Acknowledgements TARGET-DERM is a collaboration among academic and community investigators, the pharmaceutical industry, and IMISC patient community advocates. We thank the study staff, nurses, health care providers, and participants at each study center for their contributions to this work.

Contributors KA, JMC and EG-Y: substantial contributions to conception or design of the work, drafting of the work or revising it critically, final approval of the version to be published and agreement to be accountable for all aspects of the work. JIS ELS, ASP, LE, RB and DT: substantial contributions to conception or design of the work, drafting of the work or revising it critically, final approval of the version to be published. JK and JH: substantial contributions to conception or design of the work final approval of the version to be published. LD and SEW: substantial contributions to the conception or design of the work, critical revisions, final approval of the version to be published.

Funding TARGET PharmaSolutions is the sponsor and funding agency for this study. TARGET receives multisponsor support for these activities.

Map disclaimer The depiction of boundaries on this map does not imply the expression of any opinion whatsoever on the part of BMJ (or any member of its group) concerning the legal status of any country, territory, jurisdiction or area or of its authorities. This map is provided without any warranty of any kind, either express or implied.

Competing interests KA: receives compensation for consulting services from TARGET PharmaSolutions; no other competing interests. JIS: AbbVie, Anaptysbio, Arena, Asana, Boehringer-Ingelheim, Dermira, Dermavant, DS Biopharma, Eli Lilly, Galderma, GlaxoSmithKline, Glenmark, Incyte, Kiniksa, LEO Pharma, Luna, Menlo, Novartis, Pfizer, Regeneron Pharmaceuticals, Sanofi - consultant or advisory board member; Regeneron Pharmaceuticals, Sanofi - speaker. ELS: grants and personal fees from AbbVie, grants and personal fees from Eli Lilly, grants from Galderma, grants from Kyowa Hakko Kirin, grants and personal fees from Leo Pharmaceutical, grants from Merck, grants and personal fees from Pfizer, grants and personal fees from Regeneron, personal fees from Sanofi, personal fees from Dermira, grants from Galderma, grants and personal fees from Medlmmune, grants from Novartis, grants from Tioga, grants from Celgene, personal fees from Boehringer-Ingelheim, personal fees from Dermavant, personal fees from Forte Bio, personal fees from Incyte, personal fees from Menlo Therapeutics, personal fees from Ortho Dermatologics, personal fees from Pierre Fabre Dermo Cosmetique, personal fees from Valeant. ASP: investigator for AbbVie, Anaptysbio, Celgene, Eli Lilly, Galderma, Incyte, Leo, Janssen, Novartis, and Regeneron; consultant with honorarium for Almirall, Amgen, Asana, Boehringer-Ingelheim, Castle Creek, Celgene, Dermavant, Dermira, Eli Lilly, Exicure, Forte, Galderma, Lenus, Leo, MEDA Corp, Meiji Seika, Novan, Novartis, Pfizer, Regeneron, Sanofi-Genzyme, and Sol Gel. LE: Consultant/Speaker/Advisory Board: Almirall, Dermavant, Dermira, DS Biopharma, Forte, Galderma, Incyte, LEO, Lilly, L'Oreal, Matrisys, Otsuka, Novartis, Ortho Dermatologics/Valeant, Pfizer/Anacor, Regeneron, Sanofi-Genzyme. Investigator: Abvie, LEO, Regeneron, Sanofi-Genzyme. DSM: Asana, Ichnos/ Glenmark. RB: Advisory Board Member, Consultant, Speaker, Investigator for and/ or receives honoraria or grant from AbbVie, AntibioTx, Arcutis, Arena Pharma, Asana BioSciences, Bellus Health, Boehringer-Ingelheim, Dermavant, Eli Lilly, EMD Serono, Galderma, Incyte, Kiniksa, Kyowa Kirin, Neokera, LEO Pharma, Novan, Pfizer, Ralexar, RAPT, Regeneron, Sanofi Genzyme and Sienna. Employee and shareholder of Innovaderm Research. JK: Personal fees from Novartis,
Pfizer, Amgen, Lilly, Boehringer, BMS, Biogenldec, Janssen, AbbVie, Leo Pharma, ESCALIER, Valeant, Allergan, Aurigene, Sienna, UCB, Allergan, Asana, Celgene, Nimbus, Menlo, Aristea, Sanofi, Sun Pharma, Almirall, Arena, Ventyx, Aclaris, Galapagos. Grants paid to Institution from Novartis, Pfizer, Amgen, Lilly, Boehringer, Innovaderm, BMS, Janssen, AbbVie, Parexel, Leo Pharma, Vitae, Akros, Regeneron, Allergan, Novan, Biogen MA, Sienna, UCB, Celgene, Botanix, Incyte, Avillion, Exicure JH: consultant for Pfizer, Genzyme/Sanofi, Aclaris Therapeutics, Incyte, Theos Medicines, Sun Pharmaceuticals, LEO Pharma, Villaris Therapeutics, Dermavant, Temprian, AbbVie, Inc., Janssen, TeVido BioDevices, EMD Serono, Almirall, Boston Pharma, Sonoma Biotherapeutics, Inc., Methuselah Health, Twi Biotech, Pandion, Cogen Therapeutics, Inc., Admirx and BridgeBio. Investigator for Pfizer, Genzyme/ Sanofi, Aclaris Therapeutics, Incyte, Theos Medicines, Sun Pharmaceuticals, LE0 Pharma, Villaris Therapeutics, Dermavant, AbbVie, Inc., TeVido BioDevices, EMD Serono and Pandion. Equity in TeVido Biodevices, Rheos, Villaris Therapeutics, Inc. Scientific Founder of Villaris Therapeutics, Inc. LD: employee at TARGET PharmaSolutions. SEW: employee at TARGET PharmaSolutions. JMC: employee at TARGET PharmaSolutions. DT: is a lecturer and/or consultant for AbbVie, Almirall, Amgen, Asana Biosciences, Biogen Idec, BIOCAD, Boehringer Ingelheim, BristolMyers Squibb, Celgene, DS-Biopharma, GlaxoSmithKline, Janssen-Cilag, Kyowa Kirin, Leo Pharma, Eli Lilly, Novartis, Regeneron, Sandoz, Sanofi-Aventis and UCB, and received grants from AbbVie and Novartis (paid to institution). EGY: employee of Mount Sinai and has received research funds (grants paid to the institution) from Abbvie, Almirall, Amgen, AnaptysBio, Asana Biosciences, BoerhingerIngelhiem, Celgene, Dermavant, DS Biopharma, Eli Lilly, Galderma, Ichnos Sciences, Innovaderm, Janssen, Kiniska, Kyowa Kirin, Leo Pharma, Novan, Pfizer, Ralexar, Regeneron, Sienna Biopharma, UCB, and Union Therapeutics. EGY is also a consultant for Abbvie, Almirall, Amgen, Arena Pharmaceuticals, Asana Biosciences, AstraZeneca Biopharmaceuticals, Boerhinger-Ingelhiem, Cara Therapeutics, Celgene, Concert, DBV, Dermira, DS Biopharma, Eli Lilly, EMD Serono, Escalier, Galderma, Ichnos Sciences, Kyowa Kirin, Leo Pharma, Mitsubishi Tanabe, Pandion Therapeutics, Pfizer, RAPT Therapeutics, Regeneron, Sanofi, Sienna Biopharma, and Union Therapeutics.

Patient consent for publication Not required.

Provenance and peer review Not commissioned; externally peer reviewed.

Supplemental material This content has been supplied by the author(s). It has not been vetted by BMJ Publishing Group Limited (BMJ) and may not have been peer-reviewed. Any opinions or recommendations discussed are solely those of the author(s) and are not endorsed by BMJ. BMJ disclaims all liability and responsibility arising from any reliance placed on the content. Where the content includes any translated material, BMJ does not warrant the accuracy and reliability of the translations (including but not limited to local regulations, clinical guidelines, terminology, drug names and drug dosages), and is not responsible for any error and/or omissions arising from translation and adaptation or otherwise.

Open access This is an open access article distributed in accordance with the Creative Commons Attribution Non Commercial (CC BY-NC 4.0) license, which permits others to distribute, remix, adapt, build upon this work non-commercially, and license their derivative works on different terms, provided the original work is properly cited, appropriate credit is given, any changes made indicated, and the use is non-commercial. See: http://creativecommons.org/licenses/by-nc/4.0/.

\section{ORCID iDs}

Katrina Abuabara http://orcid.org/0000-0002-7736-6946

Jonathan I Silverberg http://orcid.org/0000-0003-3686-7805

Eric L Simpson http://orcid.org/0000-0002-8793-7087

Amy S Paller http://orcid.org/0000-0001-6187-6549

Lawrence F. Eichenfield http://orcid.org/0000-0002-2760-0474

Robert Bissonnette http://orcid.org/0000-0001-5927-6587

James Krueger http://orcid.org/0000-0002-3775-1778

John E. Harris http://orcid.org/0000-0002-7815-6430

Laura Dalfonso http://orcid.org/0000-0001-5442-9433

Julie M Crawford http://orcid.org/0000-0001-8976-8558

D Thaçi http://orcid.org/0000-0001-8513-550X

Emma Guttman-Yassky http://orcid.org/0000-0002-9363-324X

\section{REFERENCES}

1 Hanifin JM RG. Diagnostic features of atopic dermatitis. Acta Derm Venereol 1980;92:44-7.

2 Brunner PM, Silverberg JI, Guttman-Yassky E, et al. Increasing comorbidities suggest that atopic dermatitis Is a systemic disorder. J Invest Dermatol 2017;137:18-25. 
3 Weidinger S, Beck LA, Bieber T, et al. Atopic dermatitis. Nat Rev Dis Primers 2018;4:1.

4 Hay RJ, Johns NE, Williams HC, et al. The global burden of skin disease in 2010: an analysis of the prevalence and impact of skin conditions. J Invest Dermatol 2014;134:1527-34.

5 Barbarot S, Rogers NK, Abuabara K, et al. Strategies used for measuring long-term control in atopic dermatitis trials: a systematic review. J Am Acad Dermatol 2016;75:1038-44.

6 Paller AS, Kabashima K, Bieber T. Therapeutic pipeline for atopic dermatitis: end of the drought? J Allergy Clin Immunol 2017;140:633-43.

7 Charrow A, Xia FD, Joyce C, et al. Diversity in dermatology clinical trials: a systematic review. JAMA Dermatol 2017;153:193-8.

8 Murthy VH, Krumholz HM, Gross CP. Participation in cancer clinical trials: race-, sex-, and age-based disparities. JAMA 2004;291:2720-6.

9 Unger JM, Vaidya R, Hershman DL, et al. Systematic review and meta-analysis of the magnitude of structural, clinical, and physician and patient barriers to cancer clinical trial participation. J Natl Cancer Inst 2019;111:245-55.

10 Drucker AM, Ellis A, Jabbar-Lopez Z, et al. Systemic immunomodulatory treatments for atopic dermatitis: protocol for a systematic review with network meta-analysis. BMJ Open 2018;8:e023061.

11 Mishra P, Florian J, Peter J, et al. Public-Private partnership: targeting real-world data for hepatitis $\mathrm{C}$ direct-acting antivirals. Gastroenterology 2017;153:626-31.

12 Williams HC, Burney PG, Hay RJ, et al. The UK working party's diagnostic criteria for atopic dermatitis. I. Derivation of a minimum set of discriminators for atopic dermatitis. Br J Dermatol 1994;131:383-96.

13 Eichenfield LF, Tom WL, Chamlin SL, et al. Guidelines of care for the management of atopic dermatitis: section 1. diagnosis and assessment of atopic dermatitis. J Am Acad Dermatol 2014;70:338-51.

14 Eli Lilly and company. Validated investigator global assessment. used with the permission of Eli Lilly and company under a creative commons Attribution-NoDerivatives 4.0 international license, 2017.

15 Harmonising outcome measures for eczema. Available: www. homeforeczema.org

16 Spuls PI, Gerbens LAA, Apfelbacher CJ, et al. The International TREatment of ATopic Eczema (TREAT) registry Taskforce: an initiative to harmonize data collection across national atopic eczema photo- and systemic therapy registries. J Invest Dermatol 2017;137:2014-6.

17 Singh J. International Conference on harmonization of technical requirements for registration of pharmaceuticals for human use. J Pharmacol Pharmacother 2015;6:185-7.

18 Lindquist M. VigiBase, the WHO global ICSR database system: basic facts. Drug Inf J 2008;42:409-19.

19 Paller AS, Fölster-Holst R, Chen SC, et al. No evidence of increased cancer incidence in children using topical tacrolimus for atopic dermatitis. J Am Acad Dermatol 2020;83:375-81.

20 Margolis DJ, Abuabara K, Hoffstad OJ, et al. Association between malignancy and topical use of pimecrolimus. JAMA Dermatol 2015;151:594-9.
21 National Jewish Health. Atopic dermatitis research network (ADRN). Available: https://www.nationaljewish.org/research-science/clinicaland-translational-research/adrn/overview

22 Bosma AL, Spuls PI, Garcia-Doval I, et al. Treatment of atopic eczema (treat) registry Taskforce: protocol for a European safety study of dupilumab and other systemic therapies in patients with atopic eczema. Br J Dermatol 2020;182:1423-9.

23 Papp KA, Strober B, Augustin M, et al. PSOLAR: design, utility, and preliminary results of a prospective, international, diseasebased registry of patients with psoriasis who are receiving, or are candidates for, conventional systemic treatments or biologic agents. J Drugs Dermatol 2012;11:1210-7.

24 Eissing L, Rustenbach SJ, Krensel M, et al. Psoriasis registries worldwide: systematic overview on registry publications. J Eur Acad Dermatol Venereol 2016;30:1100-6.

25 DiMarco G, Hill D, Feldman SR. Review of patient registries in dermatology. J Am Acad Dermatol 2016;75:824-9.

26 Williams HC, Burney PG, Pembroke AC, et al. The UK working party's diagnostic criteria for atopic dermatitis. III. Independent hospital validation. Br J Dermatol 1994;131:406-16.

27 Gaunt DM, Metcalfe C, Ridd M. The patient-oriented eczema measure in young children: responsiveness and minimal clinically important difference. Allergy 2016;71:1620-5.

28 Lewis-Jones MS, Finlay AY. The children's dermatology life quality index (CDLQI): initial validation and practical use. Br J Dermato 1995;132:942-9.

29 Spuls PI, Gerbens LAA, Simpson E, et al. Patient-oriented eczema measure (POEM), a core instrument to measure symptoms in clinical trials: a Harmonising outcome measures for eczema (home) statement. Br J Dermatol 2017;176:979-84.

30 Silverberg JI, Lai J-S, Kantor RW, et al. Development, validation, and interpretation of the PROMIS itch questionnaire: a patient-reported outcome measure for the quality of life impact of itch. J Invest Dermatol 2020;140:986-94.

31 Ferreira-Valente MA, Pais-Ribeiro JL, Jensen MP. Validity of four pain intensity rating scales. Pain 2011;152:2399-404.

32 Martin S, Chandran A, Zografos L, et al. Evaluation of the impact of fibromyalgia on patients' sleep and the content validity of two sleep scales. Health Qual Life Outcomes 2009;7:64.

33 Cappelleri JC, Bushmakin AG, McDermott AM, et al. Psychometric properties of a single-item scale to assess sleep quality among individuals with fibromyalgia. Health Qual Life Outcomes 2009;7:54.

34 Health measures. Available: http://www.healthmeasures.net/index. php

35 Basra MKA, Fenech R, Gatt RM, et al. The dermatology life quality index 1994-2007: a comprehensive review of validation data and clinical results. Br J Dermatol 2008;159:997-1035.

36 Silverberg JI, Margolis DJ, Boguniewicz M, et al. Validation of five patient-reported outcomes for atopic dermatitis severity in adults. $\mathrm{Br}$ J Dermatol 2020;182:104-11.

37 Stalder J-F, Barbarot S, Wollenberg A, et al. Patient-oriented SCORAD (PO-SCORAD): a new self-assessment scale in atopic dermatitis validated in Europe. Allergy 2011;66:1114-21.

38 Reilly MC, Zbrozek AS, Dukes EM. The validity and reproducibility of a work productivity and activity impairment instrument. Pharmacoeconomics 1993;4:353-65. 